$\begin{array}{ccc}\text { UNIVERSITA } & \text { DEPARTMENT OF } \\ \text { DEGLISTUDI } \\ \text { DI TORINO }\end{array}$

\title{
BANKRUPTCY PROBLEMS WITH REFERENCE-DEPENDENT PREFERENCES
}

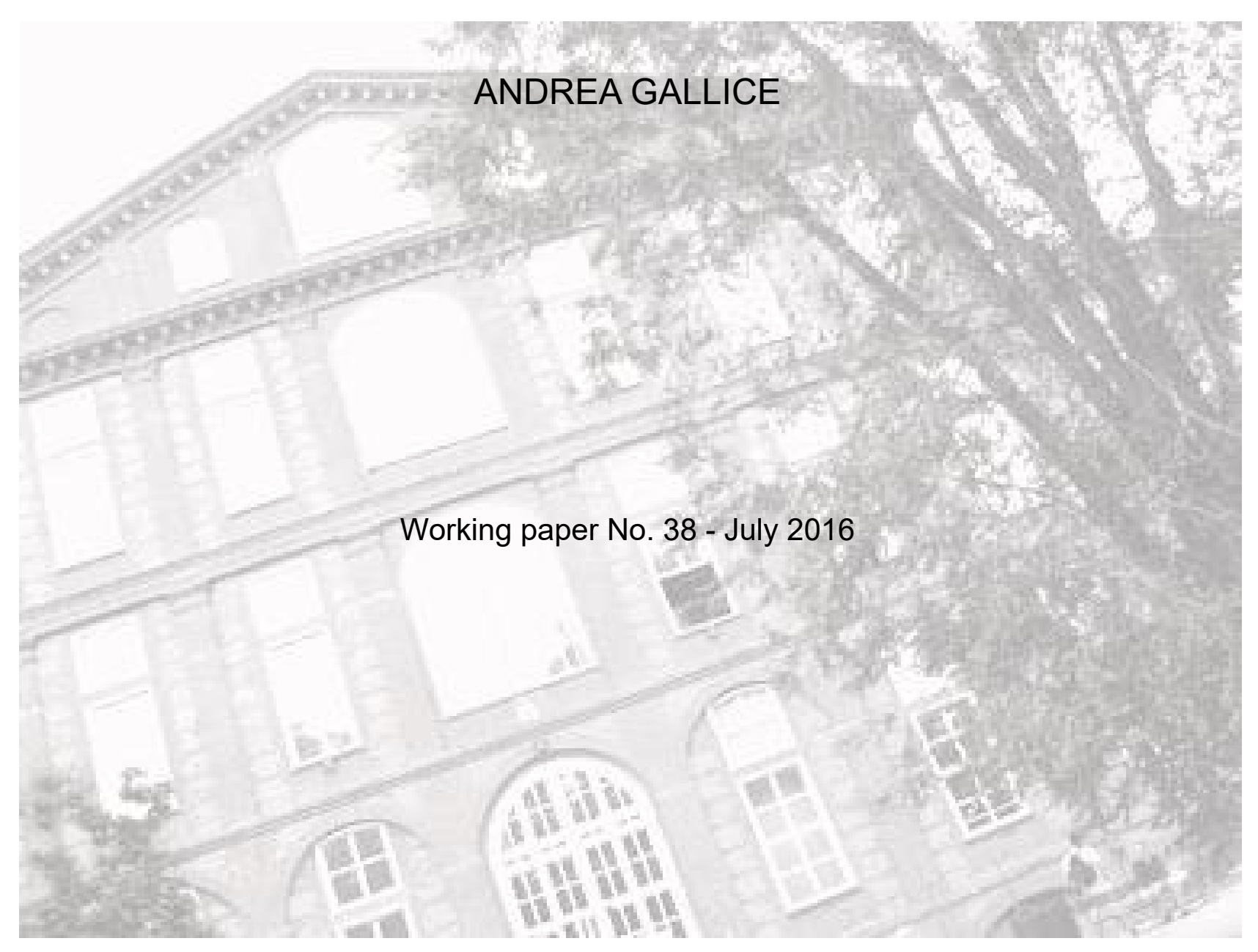




\title{
Bankruptcy problems with reference-dependent preferences
}

\author{
Andrea Gallice* \\ University of Torino and Collegio Carlo Alberto
}

\begin{abstract}
We study bankruptcy problems under the assumption that claimants have reference-dependent preferences. We show that in such a context, standard allocative rules are no longer equivalent from the viewpoint of the level of welfare that they generate. A clear ranking of the most prominent rules actually emerges. Welfare thus becomes an additional dimension that an arbitrator may want to consider in deciding which allocation to implement. We then introduce a new rule that always maximizes welfare and discuss its pros and cons.

Keywords: bankruptcy problems, claims, reference-dependent preferences, welfare. JEL classification: D63, D03.
\end{abstract}

\section{Introduction}

In a bankruptcy problem an arbitrator must allocate a finite and perfectly divisible resource among several claimants whose claims sum up to a greater amount than what is available. Real life situations that match this description include the liquidation of a bankrupted firm among different creditors, the division of an estate among a number of heirs, or the allocation of time to the completion of projects assigned by different clients.

\footnotetext{
${ }^{*}$ Contact details: ESOMAS Department, University of Torino, Corso Unione Sovietica 218bis, 10134, Torino, Italy; Collegio Carlo Alberto, Via Real Collegio 30, 10024, Moncalieri, Italy. Email: andrea.gallice@carloalberto.org. Telephone: +39 0116705287. Fax: +39 0116705082.
} 
The formal analysis of bankruptcy problems started with O'Neill (1982) and has flourished since that time (see Moulin, 2002 and Thomson, 2003, 2015 for detailed surveys). The research question that underlies this rich literature is as follows: how shall the arbitrator adjudicate conflicting claims? The answer usually takes the form of an allocative rule, i.e., a procedure that processes the data of the problem under scrutiny (namely, the total endowment of the resource and the individual claims) and then prescribes an allocation for the arbitrator to implement. The methodology through which a rule is derived can be game-theoretic (see among others Aumann and Maschler, 1985, Chun and Thomson, 1992, or Dagan and Volij, 1993) or axiomatic (Dagan, 1996, Moulin, 2000, Herrero and Villar, 2001) and different rules have different properties or respond to different ethical or procedural criteria (see Thomson, 2015, for an inventory of existing rules and relevant properties). ${ }^{1}$

A common feature of the literature is that the problem is usually investigated under the (often implicit) assumption that claimants have linear preferences. Indeed, as Thomson puts it (2015, p. 57): "In the base model, preferences are not explicitly indicated, but it is implicit that each claimant prefers more of the dividend to less." Thomson continues by saying that the base model amounts to "assuming that the utilities that claimants derive from their assignments are linear, or to ignoring utilities altogether". ${ }^{2}$ In this paper we deviate from this tradition and investigate bankruptcy problems where claimants are endowed with non-linear preferences. In particular, we focus on a specific family of preferences - so-called reference-dependent preferences (RDPs). Building upon the main insights of prospect theory (Kahneman and Tversky, 1979), RDPs (Koszegi and Rabin, 2006) explicitly acknowledge the fact that an agent's perception of a given outcome is determined not only by the outcome per se but also by how this outcome compares with a certain reference point. In other words, the agent's utility is influenced by perceived

\footnotetext{
${ }^{1}$ Thomson and Yeh, 2008, and Hougaard et al., 2012, further explore the mathematical relationships that hold between different rules by introducing the notion of "operators", i.e., mappings that associate each rule with another.

${ }^{2}$ Papers that take exception to this approach and explicitly study bankruptcy problems in a utility space are Mariotti and Villar (2005) and Herrero and Villar (2010).
} 
gains and losses. RDPs thus seem particularly appropriate for use in capturing the preferences of claimants in bankruptcy problems. These are, in fact, typical situations in which agents form their own expectations in advance about the allocation that they believe the arbitrator will implement and then inevitably compare the actual outcome with their expected one.

Indeed, the idea that reference points may play a role in bankruptcy problems is not new. Chun and Thomson (1992) study a bargaining problem with claims and interpret the disagreement point as a reference point from which agents measure their gains when evaluating a proposal. Herrero (1998) adopts a similar framework but endogenizes the reference point as a function of the agents' claims and the set of feasible allocations. Pulido et al. $(2002,2008)$ study bankruptcy problems with reference points in the context of university budgeting procedures. Finally, Hougaard et al. (2013) consider a more general model of rationing in which agents have claims as well as baselines, which can also be interpreted as reference points.

However, in line with the previous discussion, all these papers analyze the role of reference points from a context in which claimants have standard linear preferences. We instead embed the analysis of reference points into the framework of RDPs. We show that some specific features of RDPs, such as the diminishing marginal sensitivity to losses, have interesting implications and lead to novel results. In particular - and in sharp contrast to the baseline linear model - we show that when claimants display RDPs, different allocative rules are no longer equivalent from the viewpoint of welfare. Indeed, we find that the three most prominent rules (the proportional rule, the constrained equal awards rule, and the constrained equal losses rule) can be univocally ranked according to the level of welfare that they generate.

We then introduce a new rule, which we label the sequential increasing priority (sip) rule. This rule first orders the claimants on the basis of their claims, starting from the lowest (ties are broken randomly), and then assigns to each agent in turn the minimum amount between his claim and what remains of the endowment. We show that the sip rule always selects an allocation that maximizes welfare and thus dominates the other rules. More generally, the fact 
that different rules lead to different level of welfare implies that welfare emerges as an additional dimension that an arbitrator may want to consider when choosing how to adjudicate conflicting claims. Indeed, a trade-off between welfare maximization and equity may sometimes emerge, as the sip rule may fail to satisfy the "equal treatment of equals" principle. Therefore, an arbitrator who cares about equity and does not want to discriminate among equals will find the sip rule unappealing. In contrast, a more pragmatic arbitrator who aims at maximizing welfare (or equivalently, at minimizing the aggregate level of disappointment due to perceived losses) may firmly rely on the sip rule. For instance, this could be the case of a politician who must allocate limited resources among several lobbyists. In a situation of this kind, the bottom line of the paper is very clear: if claimants have reference-dependent preferences and the arbitrator wants to minimize the aggregate level of disappointment, it is better to satisfy as many claimants as possible (i.e., allocate them an amount that aligns with their claims) and give maximum disappointment to those claimants who can be disappointed the most, rather than to slightly disappoint all of the claimants.

\section{The model}

\subsection{A bankruptcy problem}

We formally define a bankruptcy problem by using the standard notation (see, among others, Dagan and Volij, 1993, Herrero and Villar, 2001, and Thomson, 2003 and 2015). Let $E \in \mathbb{R}_{+}$ denote the endowment of the resource to be allocated and $N=\{1, \ldots, n\}$ be the set of claimants. Each claimant $i \in N$ has a claim $c_{i} \in \mathbb{R}_{+}$on $E$, such that the vector $c=\left(c_{1}, \ldots, c_{n}\right)$ collects individual claims. We define as a bankruptcy problem (or claims problem) a pair $(c, E) \in \mathbb{R}_{+}^{N} \times \mathbb{R}_{+}$ where $c$ is such that $\sum_{i} c_{i} \geq E$. We denote with $\Gamma^{N}$ the class of all such problems. A rule $\tau$ is a function that associates to any problem $(c, E) \in \Gamma^{N}$ a unique award vector $x^{\tau}=\left(x_{1}^{\tau}, \ldots, x_{n}^{\tau}\right)$. The vector $x^{\tau}$ must satisfy the following basic properties: non-negativity (no claimant is asked 
to pay: $x_{i}^{\tau} \geq 0$ for any $i \in N$ ); claims boundedness (no claimant receives more than his claim: $x_{i}^{\tau} \leq c_{i}$ for any $i \in N$ ); and balance (the entire endowment is distributed: $\sum_{i} x_{i}^{\tau}=E$ ).

The literature on bankruptcy problems has characterized a large number of alternative rules that respond to different ethical or procedural criteria (Thomson, 2015). In this paper, we focus on the three most prominent rules: ${ }^{3}$

- The proportional rule (prop), which allocates the endowment proportional to the claims:

$$
\left.x_{i}^{\text {prop }}=\lambda^{\text {prop }} c_{i} \text { with } \sum_{i} \lambda^{\text {prop }} c_{i}=E \quad \text { (i.e., } \lambda^{\text {prop }}=E / \sum_{i} c_{i}\right)
$$

- The constrained equal awards rule (cea), which assigns equal awards to all claimants subject to the requirement that no one receives more than his claim:

$$
x_{i}^{c e a}=\min \left\{c_{i}, \lambda^{c e a}\right\} \text { with } \sum_{i} \min \left\{c_{i}, \lambda^{c e a}\right\}=E
$$

- The constrained equal losses rule (cel), which assigns an equal amount of losses to all claimants subject to the requirement that no one receives a negative amount:

$$
x_{i}^{c e l}=\max \left\{0, c_{i}-\lambda^{c e l}\right\} \text { with } \sum_{i} \max \left\{0, c_{i}-\lambda^{c e l}\right\}=E
$$

As it has been repeatedly noted (see, for instance, Herrero and Villar, 2001, or Gächter and Riedl, 2006), all three rules have an egalitarian flavor. In particular, the prop rule implements equality across all claimants in terms of the ratio between awards and claims. The cea rule aims at implementing the most equal allocation in terms of awards (conditional on compliance

\footnotetext{
${ }^{3}$ The prominence of these rules stems from two channels. From a theoretical perspective, these are the unique rules that simultaneously satisfy the "equal treatment of equals" property as well as the four basic axioms of scale invariance, composition, path-independence, and consistency (see Moulin, 2000, or Herrero and Villar, 2001, for more details). Moreover, these are also the rules that are most commonly used in practice (Gächter and Riedl, 2006).
} 
with the claims boundedness property). The cel rule aims at an equal distribution of losses (conditional on compliance with the non-negativity property).

The following example illustrates the solutions prescribed by these three rules in the context of a specific bankruptcy problem.

Example 1 Consider the problem $(c, E)$ with $c=(0.3,0.5,0.8)$ and $E=1$. The three rules select the following award vectors: $x^{\text {prop }}=(0.1875,0.3125,0.5), x^{\text {cea }}=(0.3,0.35,0.35)$, and $x^{c e l}=(0.1,0.3,0.6)$.

\subsection{Claimants' preferences}

Our main departure from the baseline model of a bankruptcy problem consists in explicitly modeling the claimants' preferences. In particular, we deviate from the (often implicit) assumption that claimants have linear preferences (see Thomson, 2015). Instead, we postulate that claimants display reference-dependent preferences. As discussed in the Introduction, this family of preferences seems particularly apt within the context of bankruptcy problems. Indeed, these are tipical situations in which an agent's perception of the final outcome (i.e., the award that the agent obtains from the arbitrator) depends not only on the outcome per se but also on how this outcome compares with the reference level that the agent previously had in mind.

More formally, and in line with the influential formulation proposed by Koszegi and Rabin (2006), we endow claimants with the following utility function:

$$
u_{i}\left(x_{i} \mid c_{i}\right)=x_{i}+\mu\left(x_{i}-c_{i}\right)
$$

The utility that the agent enjoys from the possession/consumption of what he obtains from the arbitrator is thus still linear, as it is usually assumed to be in the baseline model. However, the agent's overall utility is now also influenced by the "universal gain-loss function" $\mu(\cdot)$ which captures the additional effects that perceived gains or losses with respect to the agent's reference 
point have on the agent's utility. In particular, we postulate that an agent's reference point is given by his claim $c_{i}$. In other words, $c_{i}$ can be interpreted as an expression of the agent's rights, needs, demands, or aspirations (Mariotti and Villar, 2005).

The function $\mu(\cdot)$ is assumed to satisfy the following properties:

$\mathrm{P} 1: \mu(z)$ is continuous for all $z$, strictly increasing and such that $\mu(0)=0$.

P2: $\mu(z)$ is twice differentiable for $z \neq 0$.

P3: $\mu^{\prime \prime}(z)>0$ if $z<0$ and $\mu^{\prime \prime}(z)<0$ if $z>0$.

P4: if $y>z>0$ then $\mu(y)+\mu(-y)<\mu(z)+\mu(-z)$.

P5: $\lim _{z \rightarrow 0^{-}} \mu^{\prime}(z) / \lim _{z \rightarrow 0^{+}} \mu^{\prime}(z) \equiv \lambda>1$.

The $\mu(\cdot)$ function thus displays a kink when $z=0$, i.e., when the award $x_{i}$ matches the claim

$c_{i}$. Property P3 then indicates that $\mu(\cdot)$ is convex for values of $x_{i}$ that are below $c_{i}$ (domain of losses) and concave for values of $x_{i}$ that are above $c_{i}$ (domain of gains). The same property also implies that the marginal influence of these perceived gains and losses is decreasing. Property P4 means that, for large absolute values of $z$, the function $\mu(\cdot)$ is more sensitive to losses than to gains. P5 implies the same result for small values of $z: \mu(\cdot)$ is steeper when approaching the reference point from the left (losses) than when approaching from the right (gains). Taken together, these last two properties capture the loss aversion phenomenon, namely the fact that losses loom larger than gains (Kahneman and Tversky, 1979).

\subsection{Social welfare}

We are interested in studying how different allocative rules perform from a social welfare point of view. As a measure of welfare, we rely on the notion of utilitarian welfare, which is the most widely used of the welfarist approaches (for a discussion of the pros and cons of such an approach, see Gravel and Moyes, 2013). Utilitarian welfare simply amounts to the linear sum of individual utilities. The award vector $x$ thus generates welfare $W(x)=\sum_{i} u_{i}\left(x_{i}\right)$. 
Since in our context claimants are endowed with RDPs, welfare takes the following form:

$$
W(x)=\sum_{i}\left(x_{i}+\mu\left(x_{i}-c_{i}\right)\right)=E+\sum_{i} \mu\left(x_{i}-c_{i}\right)
$$

where the condition $\sum_{i} x_{i}=E$ follows directly from the fact that any allocation is required to satisfy the balance property (see Section 2.1). Expression 1 immediately shows that welfare depends on the specific allocation $x=\left(x_{1}, \ldots, x_{n}\right)$ implemented by the arbitrator. As such, aggregate welfare is no longer constant across rules. This is in sharp contrast with the baseline model, where claimants are assumed to have linear preferences. ${ }^{4}$

The following proposition ranks the proportional rule, the constrained equal awards rule, and the constrained equal losses rule on the basis of the aggregate level of welfare that each rule generates. The ranking emerges because the rules differ on how they allocate aggregate loss $L=\sum_{i} c_{i}-E$ across claimants. Since the $\mu(\cdot)$ function is strictly convex in the domain of losses, these differences lead to differences in the level of welfare.

Proposition 1 Consider a bankruptcy problem $(c, E)$ and let claimants have RDPs. The following ranking then holds:

$A-W\left(x^{c e a}\right)=W\left(x^{\text {prop }}\right)=W\left(x^{c e l}\right)$ whenever $c_{i}=c_{j}$ for all $i, j \in N$.

$B-W\left(x^{c e a}\right)>W\left(x^{\text {prop }}\right)>W\left(x^{\text {cel }}\right)$ whenever $c_{i} \neq c_{j}$ for some $i, j \in N$.

Proof. In the appendix.

The following example illustrates the results of Proposition 1 in the context of a specific bankruptcy problem.

Example 2 Consider the bankruptcy problem $(c, E)$ with $c=(0.6,0.9)$ and $E=1$ and let claimants' utility functions be given by:

\footnotetext{
${ }^{4}$ Clearly, if $u_{i}\left(x_{i}\right)=x_{i}$ for every $i \in N$ then $W(x)=\sum_{i} x_{i}=E$ no matter which specific rule the arbitrator adopts.
} 


$$
u_{i}\left(x_{i} \mid c_{i}\right)=\left\{\begin{array}{ll}
x_{i}+\frac{\left(x_{i}-c_{i}\right)^{0.88}}{0.88} & \text { if } x_{i} \geq c_{i} \\
x_{i}-2.25 \frac{\left|x_{i}-c_{i}\right|^{0.88}}{0.88} & \text { if } x_{i}<c_{i}
\end{array} \quad \text { for } i \in\{1,2\} .^{5}\right.
$$

The utilitarian social welfare function is thus given by

$$
W(x)= \begin{cases}1-2.25 \frac{\left|x_{1}-0.6\right|^{0.88}}{0.88}+\frac{\left(1-x_{1}-0.9\right)^{0.88}}{0.88} & \text { if } x_{1} \in[0,0.1] \\ 1-2.25 \frac{\left|x_{1}-0.6\right|^{0.88}}{0.88}-2.25 \frac{\left|1-x_{1}-0.9\right|^{0.88}}{0.88} & \text { if } x_{1} \in(0.1,0.6) \\ 1+\frac{\left(x_{1}-0.6\right)^{0.88}}{0.88}-2.25 \frac{\left|1-x_{1}-0.9\right|^{0.88}}{0.88} & \text { if } x_{1} \in[0.6,1]\end{cases}
$$

Since $x^{\text {prop }}=(0.4,0.6), x^{\text {cea }}=(0.5,0.5)$, and $x^{\text {cel }}=(0.35,0.65)$, it follows that $W\left(x^{\text {prop }}\right)=$ $-0.507, W\left(x^{c e a}\right)=-0.479$, and $W\left(x^{c e l}\right)=-0.510$. Coherently with Proposition 1 , the ranking $W\left(x^{\text {cea }}\right)>W\left(x^{\text {prop }}\right)>W\left(x^{\text {cel }}\right)$ thus holds. Figure 1 below illustrates the claimants' utility functions while Figure 2 depicts the social welfare function $W(x)$.

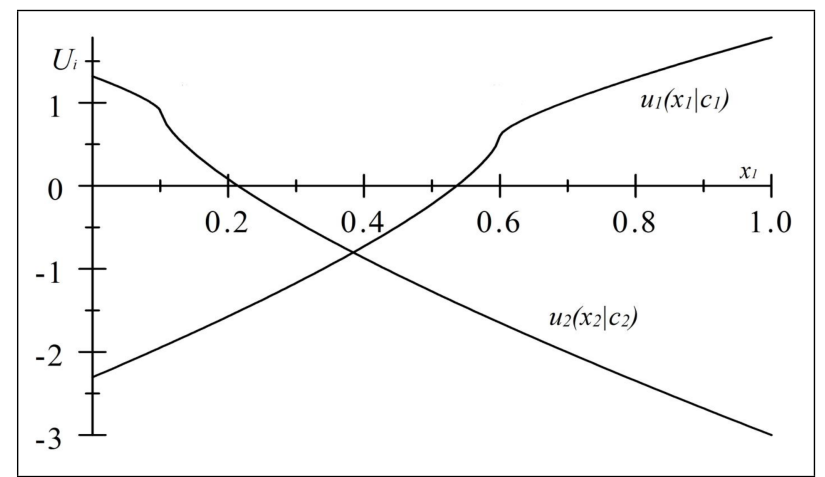

Figure 1. Claimants' utility functions. ${ }^{6}$

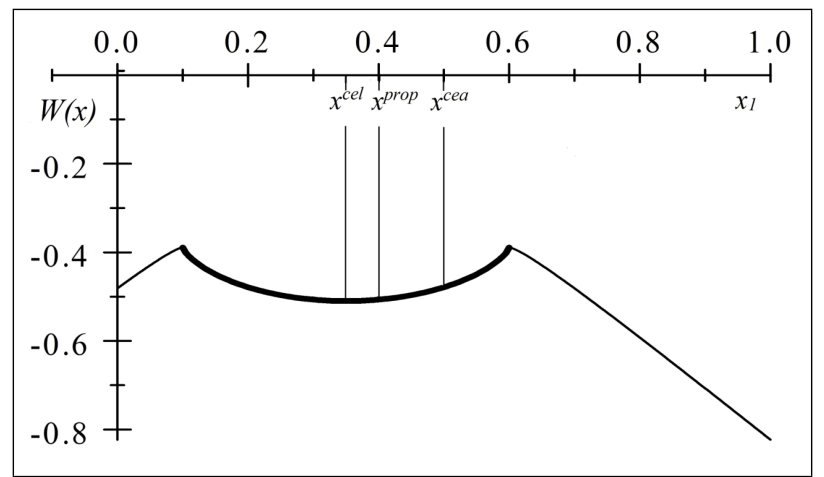

Figure 2. The utilitarian social welfare function.

\footnotetext{
${ }^{5}$ Claimants thus display the so called power gain-loss $\mu(\cdot)$ function (Tversky and Kahneman, 1992), whose general form is given by:

$$
\mu(z)= \begin{cases}\frac{z^{1-\xi}}{1-\xi} & \text { if } z \geq 0 \\ -\gamma \frac{|z|^{1-\xi}}{1-\xi} & \text { if } z<0\end{cases}
$$

and the parameters $\xi \in[0,1)$ and $\gamma>1$ determine the degree of diminishing sensitivity and the degree of loss aversion, respectively. In line with experimental evidence, and following Tversky and Kahneman (1992), we set $\xi=0.12$ and $\gamma=2.25$.

${ }^{6}$ Notice that in the figure $u_{1}\left(x_{1} \mid c_{1}\right)$ goes from left to right whereas $u_{2}\left(x_{2} \mid c_{2}\right)$ with $x_{2}=1-x_{1}$ goes from right to left.
} 


\subsection{The sequential increasing priority rule}

Proposition 1 ranks the three most prominent rules according to the level of utilitarian welfare that each rule generates. However, the proposition remains silent on the subject of how these solutions compare with the first-best solution, i.e., a solution that maximizes welfare. In this respect, Example 2 (see in particular Figure 2) suggests that the three rules do not necessarily select award vectors that maximize welfare. The intuition is that, in the context of RDPs, these rules fail to properly take into account the diminishing sensitivity to losses shown by the $\mu(\cdot)$ function. This property implies that, from a utilitarian point of view, it is more efficient to satisfy only some of the agents (i.e., allocate them an award that equals their claims) and greatly disappoint the remaining ones rather than to slightly disappoint all of the agents.

We now introduce a new rule that always selects an award vector that maximizes utilitarian welfare. We label it sequential increasing priority rule.

- The sequential increasing priority (sip) rule first orders the claimants on the basis of their claims, starting from the lowest (i.e., $c_{1} \leq c_{2} \leq \ldots \leq c_{n}$; any ties are broken randomly), and then assigns to each agent in turn the minimum amount between his claim and what remains of the endowment:

$$
x_{i}^{s i p}=\min \left\{c_{i}, \max \left\{E-\sum_{j<i} c_{j}, 0\right\}\right\}
$$

By construction, the sip rule thus selects an allocation that matches the claims of as many claimants as possible and disappoints the remaining claimants at the maximum level. ${ }^{7}$ Put differently, the sip rule selects the award vector that causes the greatest unhappiness to the least

\footnotetext{
${ }^{7}$ In the context of Example 1, the sip rule selects the allocation $x^{\text {sip }}=(0.3,0.5,0.2)$ and thus attributes the entire aggregate loss of 0.6 to agent 3 . In the context of Example 2, the sip rule selects $x^{\text {sip }}=(0.6,0.4)$ and thus attributes the entire loss to agent 2. More generally, the sip rule qualifies as a specific case of the family of sequential priority rules (see Moulin, 2000, or Thomson, 2015).
} 
number of claimants. The rule thus implements the same principle that Bossert and Suzumura (2016) characterize in a framework of ordinally measurable and interpersonally non-comparable utilities.

The following proposition shows that the sip rule achieves maximal welfare.

Proposition 2 The sip rule selects an award vector that maximizes utilitarian welfare.

Proof. In the appendix.

Three comments are in order here. First, the claim that the sip rule achieves maximal welfare holds within the domain of all those allocations that satisfy the claims boundedness property, i.e., all allocations such that $x_{i} \leq c_{i}$ for all $i \in N{ }^{8}$ Second, the fact that $x^{\text {sip }}$ maximizes utilitarian welfare is equivalent to saying that $x^{\text {sip }}$ minimizes $\sum_{i} \mu\left(x_{i}-c_{i}\right)$ (see Expression 1$)$. In other words, the sip rule allocates the aggregate loss in a way that minimizes the aggregate level of disappointment stemming from agents' perceived losses. Third, the award vector $x^{\text {sip }}$ may not be the sole welfare maximizing allocation. ${ }^{9}$ However, whenever multiple solutions exist, the sip rule will always select the award vector that is characterized by the lowest level of inequality. Therefore, an arbitrator with lexicographic preferences defined over utilitarian welfare and equality will prefer the allocation $x^{\text {sip }}$ over any other allocation. The following example illustrates this point.

\footnotetext{
${ }^{8}$ The existence of an allocation $x^{*}$ with $x_{i}^{*}>c_{i}$ for some $i \in N$ and such that $W\left(x^{*}\right)>W\left(x^{s i p}\right)$ depends on the specific shape of the agents' utility functions. In particular, $x^{*}$ exists if and only if the decrease in aggregate welfare associated with further disappointing the residual claimant $\tilde{\imath}$ by allocating him $x_{\tilde{\imath}}^{\text {sip }}-\epsilon$ is more than compensated by the increase stemming from redistributing the amount $\epsilon$ among the claimants $i=\{1, \ldots, \tilde{\imath}-1\}$. More formally, $x^{*}$ exists if and only if $(\tilde{\imath}-1) \mu\left(\frac{\epsilon}{\tilde{\imath}-1}\right)>\left[\mu\left(x_{\tilde{\imath}}^{\text {sip }}-c_{\tilde{\imath}}\right)-\mu\left(x_{\tilde{\imath}}^{\text {sip }}-\epsilon-c_{\tilde{\imath}}\right)\right]$.

${ }^{9}$ Let $c_{1}<c_{2}<\ldots<c_{n}$. The number of welfare maximizing allocations is then given by $\left|\tilde{N}_{k}\right|$ where $\tilde{N}_{k}$ is the first non-empty set $N_{k}=\left\{i \in N: c_{i} \geq L_{k}\right\} \backslash\{i \in N\}_{i=n-k+1}^{n}$ with $k \in\{0, \ldots, n-1\}, L_{k}=L-\sum_{n-k+1}^{n} c_{i}$ is the residual loss, and $L=\sum_{i} c_{i}-E$ is the aggregate loss. Clearly, the number of solutions may further increase when some of the claimants are symmetric. Notice also that when there are only two claimants and $\max \left\{c_{1}, c_{2}\right\} \leq E$ then there always exist two solutions since the condition $c_{i} \geq L$ holds for any $i \in\{1,2\}$ (see Figure 2 in Example 2 for a graphical illustration of such a situation).
} 
Example 3 Consider a bankruptcy problem $(c, 1)$ with 4 claimants and $c=(0.1,0.2,0.5,0.6)$. The sip rule selects the allocation $x^{\text {sip }}=(0.1,0.2,0.5,0.2)$ with $W\left(x^{\text {sip }}\right)=1+\mu(-0.4)$. The allocation $x^{\prime}=(0.1,0.2,0.1,0.6)$ generates the same level of welfare, $W\left(x^{\prime}\right)=1+\mu(-0.4)$. However, $x^{\text {sip }}$ implements a less unequal distribution: the Gini index of $x^{\text {sip }}$ amounts to $G\left(x^{\text {sip }}\right)=0.3$ whereas $x^{\prime}$ is such that $G\left(x^{\prime}\right)=0.4$.

Combining the results of propositions 1 and 2, we can thus present a complete ranking of the proportional rule, the constrained equal awards rule, the constrained equal losses rule, and the sequential increasing priority rule.

Proposition 3 Consider a bankruptcy problem $(c, E)$ and let claimants have RDPs. The following ranking then holds:

$A-W\left(x^{s i p}\right)>W\left(x^{\tau}\right)$ for any $\tau \in\{$ prop, cea, cel $\}$ whenever $c_{i}=c_{j}$ for all $i, j \in N$.

$B-W\left(x^{s i p}\right) \geq W\left(x^{c e a}\right)>W\left(x^{\text {prop }}\right)>W\left(x^{c e l}\right)$ whenever $c_{i} \neq c_{j}$ for some $i, j \in N$.

Proof. In the appendix.

Proposition 3 shows that the sip rule (weakly) dominates all other rules from the point of view of utilitarian welfare. On the other hand, it is important to notice that the sip rule may fail to satisfy the "equal treatment of equals" principle, which states that agents with identical claims should be treated identically. ${ }^{10}$ The "equal treatment of equals" principle is often regarded as a basic ethical property that an allocative rule should satisfy. ${ }^{11}$ Therefore an arbitrator may face a trade-off between welfare maximization and equity. ${ }^{12}$ In this respect, the sip rule does not

\footnotetext{
${ }^{10}$ This is evident in the case where all claimants are symmetric. Indeed, if $c_{i}=c_{j}$ for all $i, j \in N$, then $x^{\tau}=\left(\frac{E}{n}, \ldots, \frac{E}{n}\right)$ for any $\tau \in\{$ prop, cea,$c e l\}$, whereas $x^{s i p}=\left(c_{i}, c_{i}, \ldots, E-\sum_{j<i} c_{j}, 0, \ldots, 0\right)$.

${ }^{11}$ Concerning the four basic invariance axioms (Moulin, 2000, Herrero and Villar, 2001), it is immediate to verify that the sip rule satisfies the scale invariance axiom, the composition axiom, and the consistency axiom but fails to satisfy the path-independence axiom.

${ }^{12}$ Notice that in some circumstances the sip rule does satisfy the "equal treatment of equals" property. Say that $c=(0.3,0.3,0.3,0.4)$ and $E=1$; then, $x^{\text {sip }}=(0.3,0.3,0.3,0.1)$ such that claimants 1,2 , and 3 are treated identically.
} 
appear to be palatable for an arbitrator who wants to be impartial and and give symmetrical treatment to agents with the same claims.

However, there are situations in which the arbitrator should indeed discriminate across agents, even though their claims are symmetric (see Moulin, 2000). In these circumstances, the sip rule seems appropriate to guide the choice of a "pragmatic" arbitrator who wants to minimize the aggregate level of disappointment (i.e., the total impact that perceived losses have on welfare) across claimants. For instance, consider the case of a politician who must distribute a scarce resource (say, public funds) across several claimants (say, different associations). Assume moreover that the probability that these associations will support the politician in the next election decreases with their level of disappointment. Our analysis shows that in such a context it is wiser for the politician to fully satisfy the requests of as many associations as possible and disappoint the remaining associations at the maximum level rather than to partially disappoint all of them. Similarly, consider the situation of an agent who must allocate a limited amount of time to the completion of several tasks required by different principals. Our analysis again suggests that instead of partially progressing in the completion of every task, it is better for the agent to fully complete as many tasks as possible while totally ignoring the rest.

\section{Conclusions}

We studied bankruptcy problems under the assumption that claimants display reference-dependent preferences and thus do not only care about the award that they get but also about how this award compares with their initial claims. Contrary to the baseline model with linear utilities, we showed that in such a context, standard rules are no longer equivalent from the viewpoint of welfare. Welfare thus emerges as an additional dimension that an arbitrator may want to consider in choosing which rule to adopt. In this respect, we provided a complete ranking of the three most prominent rules (the proportional rule, the constrained equal awards rule, and the constrained 
equal losses rule) based on the level of welfare that they generate. We then introduced a new rule (the sequential increasing priority rule) that always achieves maximal welfare. However, we also discussed some potential drawbacks of the sequential increasing priority rule and showed that in some circumstances a trade-off between welfare maximization and the equal treatment of symmetric claimants may emerge. As such, we claimed that the sequential increasing priority rule appears to be appropriate for solving bankruptcy problems in which the arbitrator wants to minimize claimants' aggregate level of disappointment, and thereby minimize the number of complaints or the severity of the retaliation that he may face.

\section{Appendix: Proofs of the propositions}

\section{Proof of Proposition 1}

Case A: By construction, all three rules satisfy the "equal treatment of equals" principle. Therefore, $x^{\tau}=\left(\frac{E}{n}, \ldots, \frac{E}{n}\right)$ for any $\tau \in\{$ prop, cea,cel $\}$ whenever $c_{i}=c_{j}$ for all $i, j \in N$. It then follows that $W\left(x^{\text {prop }}\right)=W\left(x^{c e a}\right)=W\left(x^{c e l}\right)$.

Case B: The allocation $x^{\tau}$ with $\tau \in\{$ prop, cea, cel $\}$ generates utilitarian welfare $W\left(x^{\tau}\right)=$ $E+\sum_{i} \mu\left(l_{i}^{\tau}\right)$ where $l_{i}^{\tau}=\left(x_{i}^{\tau}-c_{i}\right)$ is the individual loss that agent $i$ suffers in $x^{\tau}$. Clearly, $l_{i}^{\tau} \leq 0$ for all $i$ and all $\tau$ given that all rules select an award vector that satisfies the claims boundedness property $\left(x_{i}^{\tau} \leq c_{i}\right.$ for all $\left.i \in N\right)$. Because of the balance property $\left(\sum_{i} x_{i}^{\tau}=E\right)$, aggregate loss can thus be expressed as $L=\sum_{i} l_{i}^{\tau}=\sum_{i}\left(x_{i}^{\tau}-c_{i}\right)=E-\sum_{i} c_{i}$ and thus does not depend on $\tau$. Therefore, the three rules only differ in how they allocate $L$ across claimants. Let $l^{\tau}=\left(l_{1}^{\tau}, \ldots, l_{n}^{\tau}\right)$ and consider first the cel rule. By construction, the cel rule allocates $L$ as equally as possible. As such, the rule selects the allocation that minimizes $\sigma^{2}(l)$, the variance of the elements of $l .{ }^{13}$ More formally, $\sigma^{2}\left(l^{c e l}\right)<\sigma^{2}\left(l^{\prime}\right)$ for any $l^{\prime}=\left(l_{1}^{\prime}, \ldots, l_{n}^{\prime}\right)$ such that $\sum_{i} l_{i}^{\prime}=L$

\footnotetext{
${ }^{13}$ In particular, $\sigma^{2}\left(l^{\text {cel }}\right)=0$ whenever the condition $c_{i} \geq\left(\sum_{i} c_{i}-1\right) / n$ for any $i$ holds, since in such a case the $c e l$ rule assigns the same individual loss $l_{i}^{\text {cel }}=\frac{L}{n}$ to every $i$. In contrast, $\sigma^{2}\left(l^{\text {cel }}\right)>0$ whenever the condition $c_{i}<\left(\sum_{i} c_{i}-1\right) / n$ for some $i$ holds since in this case an egalitarian allocation of $L$ is not feasible.
} 
and $l_{i}^{\prime} \neq l_{j}^{\prime}$ for some $i, j \in N$. The strict convexity of the $\mu(\cdot)$ function in the domain of losses then implies that $\sum_{i} \mu\left(l_{i}^{c e l}\right)<\sum_{i} \mu\left(l_{i}^{\prime}\right)<0$. Since $x^{c e l} \neq x^{\tau}$ with $\tau \in\{$ prop, cea $\}$ whenever $c_{i} \neq c_{j}$ for some $i, j \in N$, it follows that $l^{c e l} \neq l^{\tau}$ with $\tau \in\{$ prop,cea $\}$ and thus it must be the case that $\min \left\{\sigma^{2}\left(l^{\text {prop }}\right), \sigma^{2}\left(l^{c e a}\right)\right\}>\sigma^{2}\left(l^{c e l}\right)$. Therefore, $\min \left\{W\left(x^{\text {prop }}\right), W\left(x^{c e a}\right)\right\}>W\left(x^{c e l}\right)$. Now compare the cea and the prop rule. Assume first that the condition $c_{i}>\frac{E}{n}$ for all $i$ holds. The cea rule then selects the egalitarian allocation $x^{\text {cea }}=\left(\frac{E}{n}, \ldots, \frac{E}{n}\right)$. Therefore, $l^{\text {cea }}$ is such that $l_{i}^{c e a}=\frac{E}{n}-c_{i}$ for all $i$. It follows that $\sigma^{2}\left(l^{c e a}\right)=\sigma^{2}(c)$. The prop rule instead selects the allocation $x_{i}^{\text {prop }}=\lambda^{\text {prop }} c_{i}$ with $\lambda^{\text {prop }}=E / \sum_{i} c_{i}$ such that $\lambda^{\text {prop }} \in(0,1)$. Therefore, $l_{i}^{\text {prop }}=\left(\lambda^{\text {prop }}-1\right) c_{i}$ for all $i$. It follows that $\sigma^{2}\left(l^{\text {prop }}\right)=\left(\lambda^{\text {prop }}-1\right)^{2} \sigma^{2}(c)$. This implies that $\sigma^{2}\left(l^{c e a}\right)>\sigma^{2}\left(l^{\text {prop }}\right)$ given that $\left(\lambda^{\text {prop }}-1\right)^{2}<1$. If on the other hand, the condition $c_{i} \leq \frac{E}{n}$ for some $i$ holds then $l^{\text {cea }}$ is such that $l_{i}^{\text {cea }}=0$ for some $i$. It follows that $\sigma^{2}\left(l^{c e a}\right)>\sigma^{2}(c)$ and the condition $\sigma^{2}\left(l^{\text {cea }}\right)>\sigma^{2}\left(l^{\text {prop }}\right)$ thus holds a fortiori. In other words, the proportional rule leads to an allocation of individual losses that displays a lower variance with respect to the allocation that stems from applying the constrained equal awards rule. Because of the strict convexity of the $\mu(\cdot)$ function, it then follows that $W\left(x^{c e a}\right)>W\left(x^{p r o p}\right)$. We can thus complete the ranking and state that $W\left(x^{c e a}\right)>W\left(x^{p r o p}\right)>W\left(x^{c e l}\right)$.

\section{Proof of Proposition 2}

Consider the problem $\max _{x} W=E+\sum_{i} \mu\left(x_{i}-c_{i}\right)$. A solution certainly exists given that $W(\cdot)$ is continuous in the closed and bounded space defined by the conditions $x_{i} \in \mathbb{R}_{+}$for all $i \in N$ and $\sum_{i} x_{i}=E$, and thus the Weierstrass theorem applies. Any allocation $x$ that satisfies the claims boundedness property is such that $x_{i} \leq c_{i}$ for all claimants. Given that $\sum_{i} c_{i}>E$, it must then be the case that $x_{i}<c_{i}$ for $m$ claimants with $m \in\{1, \ldots, n\}$. The function $W(\cdot)$ is thus given by the sum of a constant and $m$ strictly convex functions since $\mu^{\prime \prime}(z)>0$ when $z<0$. Therefore, $W(\cdot)$ is strictly convex and the function reaches a maximum as a corner solution (i.e., at a point in which $x_{i}=c_{i}$ for some $i \in N$ ). The diminishing marginal sensitivity 
of the $\mu(\cdot)$ function implies $|\mu(0)+\mu(a+b)|<|\mu(a)+\mu(b)|$ for any $a, b<0$. Therefore, the allocations that maximize utilitarian welfare are those that assign $x_{i}=c_{i}$ to as many agents as possible and disappoint as much as possible the claimants that can be disappointed the most. By construction, the sequential increasing priority rule selects such an allocation.

\section{Proof of Proposition 3}

It is immediate to show that, whenever $c_{i}=c_{j}$ for all $i, j \in N$, the strict ranking $W\left(x^{s i p}\right)>$ $W\left(x^{\tau}\right)$ for any $\tau \in\{$ prop, cea, cel $\}$ holds. Indeed, because of the properties of the $\mu(\cdot)$ function, $W\left(x^{\text {sip }}\right)>W\left(x^{\text {prop }}\right)$ since $x^{\text {prop }}$ is such that $x_{i}^{\text {prop }}-c_{i}<0$ for all $i \in N$. Moreover, because of Proposition 1, we know that if $c_{i}=c_{j}$ for all $i, j \in N$ then $W\left(x^{\text {prop }}\right)=W\left(x^{c e a}\right)=W\left(x^{c e l}\right)$. It then follows that $W\left(x^{s i p}\right)>W\left(x^{\tau}\right)$ for any $\tau \in\{$ prop, cea, cel $\}$. Concerning case $\mathrm{B}$, the condition $W\left(x^{s i p}\right)=W\left(x^{c e a}\right)$ only holds if there exist $n-1$ claimants with $c_{i}<\frac{E}{n}$ and one claimant $j$ with $c_{j}>E-\sum_{i \neq j} c_{i}$. Indeed, it is easy to verify that in such a situation $x^{s i p}$ and $x^{c e a}$ coincide (in particular, $x_{i}^{s i p}=x_{i}^{c e a}=c_{i}$ for all $i$ and $x_{j}^{s i p}=x_{j}^{c e a}=E-\sum_{i \neq j} c_{i}$ ). In all other cases, the strict ordering $W\left(x^{s i p}\right)>W\left(x^{c e a}\right)$ holds.

\section{References}

[1] Aumann, R.J., Maschler, M., 1985. Game theoretic analysis of a bankruptcy problem from the Talmud. Journal of Economic Theory, 36, 195-213.

[2] Bossert, W., Suzumura, K., 2016. The greatest unhappiness of the least number. Social Choice and Welfare, forthcoming.

[3] Chun, Y., Thomson, W., 1992. Bargaining problems with claims. Mathematical Social Sciences, 24, 19-33.

[4] Dagan, N., 1996. New characterizations of old bankruptcy rules. Social Choice and Welfare, $13,51-59$. 
[5] Dagan, N., Volij, O., 1993. The bankruptcy problem: a cooperative bargaining approach. Mathematical Social Sciences, 26, 287-297.

[6] Gächter, S., Riedl, A., 2006. Dividing justly in bargaining problems with claims: Normative judgments and actual negotiations. Social Choice and Welfare, 27, 571-594.

[7] Gravel, N., Moyes, P., 2013. Utilitarianism or welfarism: does it make a difference? Social Choice and Welfare, 40, 529-551.

[8] Herrero, C., 1998. Endogenous reference points and the adjusted proportional solution for bargaining problems with claims. Social Choice and Welfare, 15, 113-119.

[9] Herrero, C., Villar, A., 2001. The three musketeers: four classical solutions to bankruptcy problems. Mathematical Social Sciences, 39, 307-328.

[10] Herrero, C., Villar, A., 2010. The rights egalitarian solution for NTU problems. International Journal of Game Theory, 39, 137-150.

[11] Hougaard, J.L., Moreno-Ternero, J., Østerdal, L.P., 2012. A unifying framework for the problem of adjudicating conflicting claims, Journal of Mathematical Economics, 48,107114.

[12] Hougaard, J.L., Moreno-Ternero, J., Østerdal, L.P., 2013. Rationing in the presence of baselines. Social Choice and Welfare, 40, 1047-1066.

[13] Kahneman, D., Tversky, A., 1979. Prospect theory: An analysis of decision under risk. Econometrica 47 (2), 263-291.

[14] Koszegi, B., Rabin, M., 2006. A model of reference-dependent preferences. Quarterly Journal of Economics 121 (4), 1133-1165.

[15] Mariotti, M., Villar, A., 2005. The Nash rationing problem. International Journal of Game Theory, 33, 367-377. 
[16] Moulin, H., 2000. Priority rules and other asymmetric rationing methods. Econometrica, $68,643-684$.

[17] Moulin, H., 2002. Axiomatic cost and surplus-sharing. In: Arrow, K., Sen, A., Suzumura, K. (Eds.), Handbook of Social Choice and Welfare, Vol. 1, Amsteradm: Elsevier, 543-96.

[18] O'Neill, B., 1992. A problem of rights arbitration from the Talmud. Mathematical Social Sciences, 2, 345-371.

[19] Pulido, M., Borm, P., Hendrichx, R., Llorca, N., Sanchez-Soriano, J., 2008. Compromise solutions for bankruptcy situations with references. Annals of Operations Research, 158, 133-141.

[20] Pulido, M., Sanchez-Soriano, J., Llorca, N., 2002. Game theory techniques for university management: an extended bankruptcy model. Annals of Operations Research, 109, 129-142.

[21] Thomson, W., 2003. Axiomatic and game-theoretic analysis of bankruptcy and taxation problems: A survey. Mathematical Social Sciences, 45 (3), 249-297.

[22] Thomson, W., 2015. Axiomatic and game-theoretic analysis of bankruptcy and taxation problems: An update. Mathematical Social Sciences, 74 (3), 41-59.

[23] Thomson, W., Yeh, C.-H., 2008. Operators for the adjudication of conflicting claims. Journal of Economic Theory, 143, 177-198.

[24] Tversky, A., Kahneman, D., 1992. Advances in prospect theory: Cumulative representation of uncertainty. Journal of Risk and Uncertainty, 5 (4), 297-323. 
DEPARTMENT OF ECONOMICS AND STATISTICS

UNIVERSITY OF TORINO

Corso Unione Sovietica 218 bis - 10134 Torino (ITALY)

Web page: http://esomas.econ.unito.it/ 